\title{
Selective CB2 Receptor Agonism Protects Central Neurons from Remote Axotomy-Induced Apoptosis through the PI3K/Akt Pathway
}

\author{
Maria Teresa Viscomi, ${ }^{1,2}$ Sergio Oddi, ${ }^{1,3}$ Laura Latini, ${ }^{1,2}$ Nicoletta Pasquariello, ${ }^{3}$ Fulvio Florenzano, ${ }^{1,4}$ \\ Giorgio Bernardi, ${ }^{1,5}$ Marco Molinari, ${ }^{1,2 *}$ and Mauro Maccarrone ${ }^{1,3 *}$ \\ ${ }^{1}$ Santa Lucia Foundation Istituto di Ricovero e Cura a Carattere Scientifico, 00143 Rome, Italy, ${ }^{2}$ Spinal Trauma and Disease Foundation Onlus, 37135 \\ Verona, Italy, ${ }^{3}$ Department of Biomedical Sciences, University of Teramo, 64100 Teramo, Italy, ${ }^{4}$ Confocal Microscopy Unit, European Brain Research \\ Institute/Consiglio Nazionale delle Ricerche/Santa Lucia Foundation, 00143 Rome, Italy, and ${ }^{5}$ Department of Neuroscience, University of Rome "Tor \\ Vergata," 00133 Rome, Italy
}

Endocannabinoids are neuroprotective in vivo and in vitro, but the mechanisms by which they act are largely unknown. The present study addressed the role of cannabinoid receptors during remote cell death of central neurons in a model that is based on cerebellar lesions. A lesion in one cerebellar hemisphere induced remote cell death and type 2 cannabinoid receptor (CB2R) expression in contralateral precerebellar neurons. Of the selective agonists and antagonists that modulated cannabinoid receptor activity, we found that the CB2R agonist JWH-015 reduced neuronal loss and cytochrome- $c$ release, leading to neurological recovery; these effects were reversed by the selective CB2R antagonist SR144528. Analysis of CB2R-triggered signal transduction demonstrated that in axotomized neurons, CB2R regulated Akt and JNK phosphorylation through a PI3K-dependent pathway, whereas other major signaling routes that are dependent on CB2R, such as ERK1/2 and p38, were not involved. This result was corroborated by the observation that the selective PI3K inhibitor LY294002 blocked the CB2R stimulation effects on neuronal survival as well as Akt and JNK phosphorylation levels. Together, these data demonstrate that axonal damage induces $\mathrm{CB} 2 \mathrm{R}$ expression in central neurons and that stimulation of this receptor has a neuroprotective effect that is achieved through PI3K/Akt signaling.

\section{Introduction}

Endocannabinoids have been involved in the regulation of mammalian cell apoptosis (Maccarrone and Finazzi-Agró, 2003), as well in the pathogenesis of neuroinflammatory (Klein et al., 2003) and neurodegenerative diseases (Centonze et al., 2007). Endocannabinoids are lipid signals that exert most of their actions via activation of specific G-protein-coupled receptors-the type 1 and type 2 cannabinoid receptors (CB1R and CB2R) (Di Marzo et al., 2007).

$\mathrm{CB} 1 \mathrm{R}$ is highly expressed in the brain, and CB2R initially was considered to be expressed primarily in immune and hematopoietic cells (Mackie, 2006). Recent studies, however, have documented CB2R expression in the brain in both glial and neuronal cells (Van Sickle et al., 2005; Onaivi et al., 2006).

\footnotetext{
Received Feb. 16, 2009; accepted March 2, 2009.

This investigation was partly supported by Ministero dell'Università e della Ricerca (Fondo di Incentivazione della Ricerca di Base 2006), and by Fondazione (assa di Risparmio di Teramo (Research Programs 2004 and 2005) to M. Maccarrone and by Ministero della Salute to M. Molinari and G. Bernardi. We gratefully acknowledge the support of Dr. Flavio Keller and Dr. Filippo Biamonte for the use of stereological equipment. SANOFI-AVENTIS is gratefully acknowledged for kindly providing the selective cannabinoid receptors antagonists SR141716A and SR144528. The professional editorial work of Blue Pencil Science LLC is also acknowledged.

${ }^{*} M$. Molinari and M. Maccarrone are equally senior authors.

Correspondence should be addressed to Marco Molinari, Experimental Neurorehabilitation Laboratory, Santa Lucia Foundation, Via Ardeatina 306, 00179 Rome, Italy. E-mail: m.molinari@hsantalucia.it.

DOI:10.1523/JNEUROSCI.0786-09.2009

Copyright $\odot 2009$ Society for Neuroscience $\quad$ 0270-6474/09/294564-07\$15.00/0
}

Most of the effects of endocannabinoids in the CNS are considered to be mediated by the activation of the CB1R, the role of CB2R being limited to glia-dependent anti-inflammatory actions (Stella, 2004). Moreover, the expression and function of CB2R in the brain remain controversial. A recent pharmacological MRI study concluded, however, that CB2R is not functionally active in the brain under physiological conditions (Chin et al., 2008).

Remote cell death can be defined as neuronal death that occurs in regions that are remote to the primary site of a lesion after focal brain damage (Block et al., 2005). These remote effects have been associated with inflammation (Viscomi et al., 2008) and are considered to be an important predictor of outcome (Binkofski et al., 1996). The experimental paradigm that is based on hemicerebellectomy $(\mathrm{HCb})$ is a well known and highly reproducible model that is used to study remote cell death (Viscomi et al., 2008). In this model, neuronal degeneration is induced by target deprivation and axonal damage of precerebellar neurons (Viscomi et al., 2004).

To address the role of CBR-mediated signaling in neuroprotection and neurodegeneration, we studied CB1R and CB2R expression and the effects of their modulation in response to different CBR agonists and antagonists in a neurodegenerative model of remote cell death, based on cerebellar focal lesions. Here, we show that $\mathrm{HCb}$ induces $\mathrm{CB} 2 \mathrm{R}$ expression in precerebellar neurons and that JWH-015, a selective CB2R agonist, reduces remote 
Table 1. Lesion and treatments in the different experimental groups

\begin{tabular}{|c|c|c|c|}
\hline Group codes & $n$ & $\mathrm{HCb}$ & Drug treatment \\
\hline CTRL-Eth & 16 & & Ethanol (400 $\mu \mathrm{l}, 0.5 \%$ saline, i.p., for 7 d) \\
\hline $\mathrm{HCb}$-Eth & 16 & $x$ & Ethanol ( $400 \mu \mathrm{l}, 0.5 \%$ saline, i.p., for 7 d) \\
\hline CTRL-ACEA & 5 & & ACEA (5 mg/kg, i.p., for $7 \mathrm{~d}$ ) \\
\hline $\mathrm{HCb}-\mathrm{ACEA}$ & 5 & $x$ & ACEA (5 mg/kg, i.p., for $7 \mathrm{~d}$ ) \\
\hline CTRL-SR1 & 5 & & SR141716A (3 mg/kg, i.p., for $7 \mathrm{~d}$ ) \\
\hline $\mathrm{HCb}-\mathrm{SR} 1$ & 5 & $X$ & SR141716A (3 mg/kg, i.p., for $7 \mathrm{~d}$ ) \\
\hline CTRL-JWH & 16 & & JWH-015 (3 mg/kg, i.p., for 7 d) \\
\hline HCb-JWH & 16 & $X$ & JWH-015 (3 mg/kg, i.p., for 7 d) \\
\hline CTRL-SR2 & 16 & & SR144528 (3 mg/kg, i.p., for 7 d) \\
\hline $\mathrm{HCb}-\mathrm{SR} 2$ & 16 & $X$ & SR144528 (3 mg/kg, i.p., for 7 d) \\
\hline CTRL-CAPS & 5 & & Capsaicin (1 mg/kg, i.p., for 7 d) \\
\hline $\mathrm{HCb}$-CAPS & 5 & $X$ & Capsaicin (1 mg/kg, i.p., for 7 d) \\
\hline CTRL-CAPZ & 5 & & Capsazepine (3 mg/kg, i.p., for 7 d) \\
\hline $\mathrm{HCb}-\mathrm{CAPZ}$ & 5 & $x$ & Capsazepine (3 mg/kg, i.p., for 7 d) \\
\hline CTRL-LY-Eth & 5 & & LY294002 (15 $\mu \mathrm{g} / 10 \mu \mathrm{l} ; 25 \%$ DMS0, i.c.v.) + ethanol (400 $\mu \mathrm{l}, 0.5 \%$ saline, i.p., for $7 \mathrm{~d}$ ) \\
\hline HCb-LY-Eth & 5 & $X$ & LY294002 (15 $\mu \mathrm{g} / 10 \mu \mathrm{l} ; 25 \%$ DMS0 i.c.v.) + ethanol (400 $\mu$ l, $0.5 \%$ saline, i.p., for $7 \mathrm{~d})$ \\
\hline CTRL-LY-JWH & 5 & & LY294002 (15 $\mu \mathrm{g} / 10 \mu \mathrm{l} ; 25 \%$ DMS0 i.c.v.) + JWH-015 (3 mg/kg, i.p., for 7 d) \\
\hline HCb-LY-JWH & 5 & $X$ & LY294002 (15 $\mu \mathrm{g} / 10 \mu \mathrm{l} ; 25 \%$ DMS0 i.c.v.) + JWH-015 (3 mg/kg, i.p., for 7 d) \\
\hline CTRL-SP-Eth & 5 & & SP600125 (30 $\mu \mathrm{g} / 10 \mu \mathrm{l} ; 25 \%$ DMSO i.c.v.) + ethanol (400 $\mu$ l, $0.5 \%$ saline, i.p., for $7 \mathrm{~d})$ \\
\hline HCb-SP-Eth & 5 & $X$ & SP600125 (30 $\mu \mathrm{g} / 10 \mu \mathrm{l} ; 25 \%$ DMSO i.c.v.) + ethanol ( $400 \mu \mathrm{l}, 0.5 \%$ saline, i.p., for $7 \mathrm{~d})$ \\
\hline CTRL-SP-JWH & 5 & & SP600125 (30 $\mu \mathrm{g} / 10 \mu \mathrm{l} ; 25 \%$ DMS0 i.c.v.) + JWH-015 (3 mg/kg, i.p., for 7 d) \\
\hline HCb-SP-JWH & 5 & $X$ & SP600125 (30 $\mu \mathrm{g} / 10 \mu \mathrm{l} ; 25 \%$ DMS0 i.c.v.) + JWH-015 (3 mg/kg, i.p., for $7 \mathrm{~d})$ \\
\hline
\end{tabular}

CTRL, Control.

cell death and promotes neurological recovery by stimulating the PI3K/Akt pathway.

\section{Materials and Methods}

Animals and surgery. Cerebellar lesions were attained by performing a right $\mathrm{HCb}$, as described previously (Viscomi et al., 2004).

Drug treatment. Drug treatment groups are listed in Table 1. Compounds and manufacturers are listed in the supplemental material (available at www.jneurosci.org).

Neurological evaluation. Neurological impairment was evaluated by the Neurological Severity Score (NSS) (Watanabe et al., 2004), described in detail in supplemental material (available at www.jneurosci.org).

Histology and immunohistochemistry. The complete list of the primary antibodies used as well as details of immunohistochemistry and immunofluorescence techniques are reported in supplemental material (available at www.jneurosci.org).

Protein isolation/Western blotting. This procedure was performed as described previously (Viscomi et al., 2008) and detailed in supplemental material (available at www.jneurosci.org).

Quantitative real-time PCR. This procedure is detailed in supplemental material (available at www.jneurosci.org).

Quantitative and statistical analyses. Stereological counts were performed on five Nissl/CB2R-stained sections both for the inferior olive (IO) and pontine nuclei $(\mathrm{Pn})$. Cell counts and densitometric analysis on fluorescent material were performed off-line on confocal images (for details see supplemental material, available at www.jneurosci.org). All values were expressed as mean \pm SD. Differences between means were analyzed using one-way or two-way ANOVA with a Bonferroni post hoc test. All statistical analyses were performed using Prism-4 software (GraphPad Software for Science), with significance set at $p<0.05$.

\section{Results}

We first assessed the expression of CB1R and $\mathrm{CB} 2 \mathrm{R}$ in the IO and Pn under physiological conditions by immunohistochemistry. The IO and Pn expressed CB1R but not CB2R (Fig. 1A). In addition, we failed to detect the presence of the endocannabinoidbinding vanilloid receptor-1 (TRPV1) in either the IO or Pn (data not shown).

Damage to one cerebellar hemisphere significantly reduced the number of neurons in the contralateral IO and Pn. Seven days after lesion, the number of surviving neurons was reduced to $\sim 50 \%$ of the prelesional levels (Fig. $1 B$ ).

Further, $\mathrm{HCb}$ altered the expression of cannabinoid receptors. In fact, all surviving postlesional IO and Pn neurons expressed CB1R, and $\sim 40 \%$ to $50 \%$ of them also expressed CB2R (Fig. 1A,B). Double immunofluorescence of CB2R and NeuN confirmed that CB2R was expressed by neurons (Fig. $1 C$ ), while double labeling of CB2R and GFAP or OX-42 demonstrated that astrocytic and microglial cells lacked CB2R (supplemental Fig. 1, available at www.jneurosci.org as supplemental material). TRPV1 expression was not detected after $\mathrm{HCb}$ in either the IO or Pn (data not shown).

The differential expression of CB1R and CB2R mRNA in normal and lesioned animals was assessed by quantitative realtime PCR (qPCR). Consistent with our immunostaining results, control animals expressed low levels of CB2R mRNA compared with CB1R mRNA, both in the IO (0.04-fold; $p<0.001)$ and Pn (0.3-fold; $p<0.05)$. At day 7 after lesion, CB2R mRNA levels increased 12 -fold $(p<0.001)$ and 3.2 -fold $(p<0.001)$ in the IO and Pn, respectively, compared with prelesional levels, while CB1R mRNA levels increased 0.14-fold $(p<0.01)$ in the IO and 0.68fold $(p<0.05)$ in Pn (Fig. $1 D)$. HCb was associated also with a marked downregulation in mRNA expression of enolase, a neuronal marker (Fig. 1D).

To evaluate the efficacy of CB2R induction in influencing the fate of axotomized cells, we treated control and hemicerebellectomized (HCbed) animals with different CBR agonists and antagonists chronically (Table 1).

Neuronal cell counts, performed in lesioned animals after $7 \mathrm{~d}$ of treatment, demonstrated a selective protective effect of the CB2R agonist JWH-015. Despite differences in pharmacological treatment, the cell counts of all lesioned groups were lower than those of unlesioned groups (Fig. 2A). One-way ANOVA, performed on the number of surviving neurons in the control and HCbed groups, revealed a significant effect of the lesion (IO: $F=$ $60.05, p<0.0001$; Pn: $F=67.23, p<0.0001$ ). Post hoc comparisons of both the IO and Pn data confirmed that each of the treated control groups was significantly different from all of the treated HCbed groups $(p<0.001)$.

Within the HCbed groups, JWH-015 treatment induced the highest number of surviving cells in both the IO and $\mathrm{Pn}$, while HCb-SR2 animals had the fewest such cells (Fig. 2A). Post hoc comparisons of both the IO and Pn data demonstrated that HCbJWH animals were significantly different from HCb-Eth $(p<$ $0.001)$, HCb-SR2 $(p<0.001)$, HCb-SR1 $(p<0.01)$, HCb-ACEA $(p<0.01)$, HCb-Caps $(p<0.01)$, and HCb-Capz $(p<0.01)$ animals. No significant differences were present between the HCb-Eth, HCb-SR2, HCb-ACEA, HCb-SR1, HCb-Caps, and HCb-Capz groups (Fig. 2A).

Further, by NSS only JWH-015 treatment was associated with a favorable neurological outcome. The NSSs for all groups at different time points are shown in Figure 2, $B$ and $C$. In all treated unlesioned rats, NSS values were $\sim 0$ (Fig. $2 B$ ). Twenty-four hours after $\mathrm{HCb}$, all groups, despite the variation in treatments, 

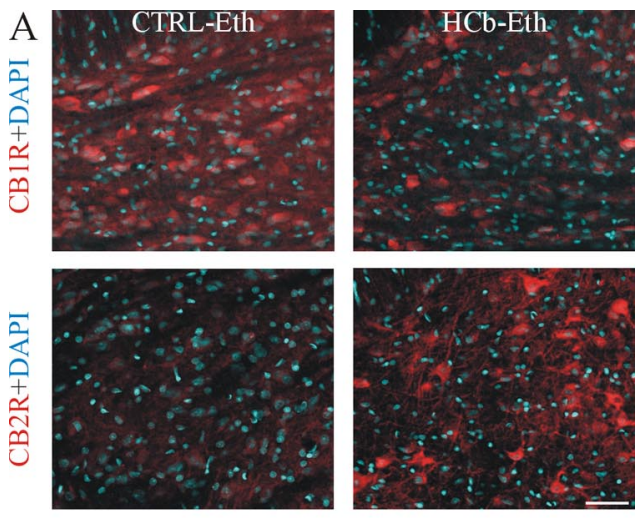

B

INFERIOR OLIVE
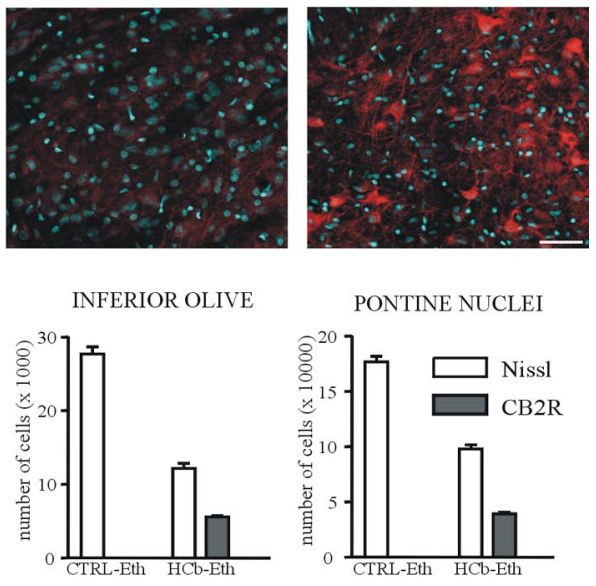

PONTINE NUCLEI
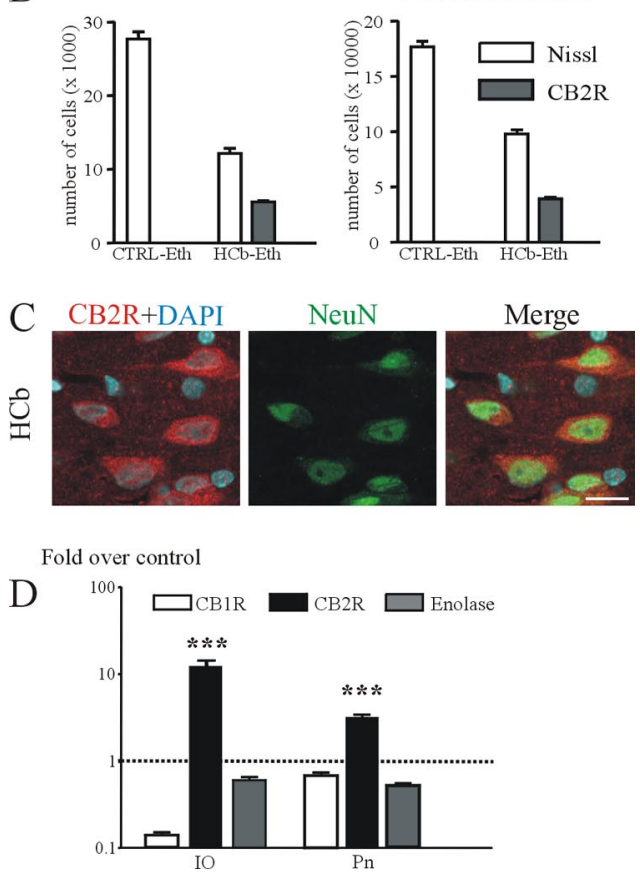

Figure 1. Hemicerebellectomy induces CB2R expression in precerebellar stations. $A$, Confocal images from the Pn of CTRL-Eth and HCb-Eth animals, reacted with CB1R and CB2R antibodies and counterstained with DAPI. B, Number of Nissl-stained and CB2R-positive neurons in the 10 and $\mathrm{Pn}$ in CTRL-Eth and HCb-Eth rats. Data are reported as means $\pm S D(n=5$ animals per group). C, Confocal images showing (B2R and NeuN immunostaining plus DAPI counterstaining. $D, C B 1 R$, CB2R, and enolase mRNA expression in the 10 and $\mathrm{Pn}$ of $\mathrm{HCb}$-Eth rats. Data are presented as fold changes in target gene expression, normalized to the internal control gene and relative to the tissue control. Data are reported as means $\pm S D(n=4$; each performed in triplicate). ${ }^{* *} p<0.0001$. Scale bars: $A, 40 \mu \mathrm{m} ; C, 10 \mu \mathrm{m}$.

generated an NSS of $\sim 9$ (Fig. $2 C$ ). At later time points, the values tended to remain constant within each group, with the exception of $\mathrm{HCb}-\mathrm{JWH}$ animals, which showed progressive improvement, and $\mathrm{HCb}-\mathrm{SR} 2$ animals, which experienced a slight decline in NSS (Fig. 2C).

Two-way ANOVA (time $\times$ treatment) demonstrated a significant overall effect of time $(F=77.07 ; p<0.0001)$ and treatment $(F=8.75 ; p<0.0001)$; moreover, their interaction was significant $(F=4.58 ; p<0.001)$. Post hoc comparisons showed that $\mathrm{HCb}-\mathrm{JWH}$ scores at $72 \mathrm{~h}$ as well as at 5 and $7 \mathrm{~d}$ were significantly different from those of all of the other groups $(p<0.001)$. No other significant differences were observed (Fig. 2C).

The positive and negative effects of JWH-015 and SR144528 treatment, respectively, on functional outcome were further corroborated by $\Delta$ NSS values (Fig. $2 D$ ). Two-way ANOVA (time $\times$
A
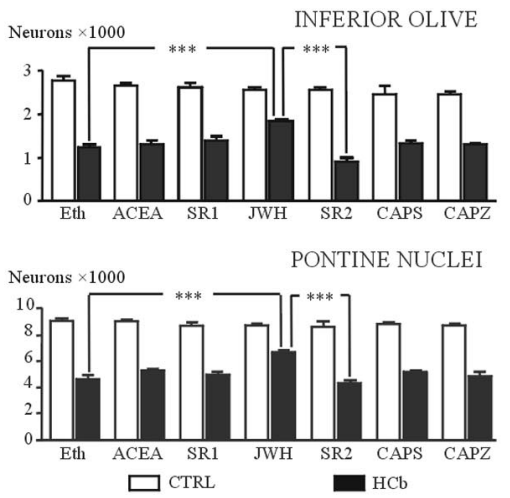

$\mathrm{B}$

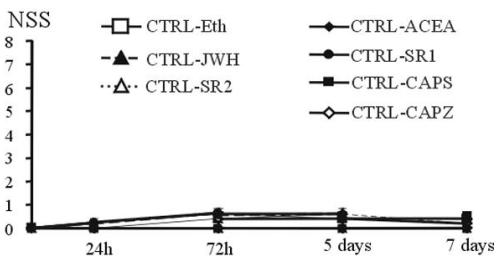

C NSS $-\square-\mathrm{HCb}-\mathrm{Eth} \quad \rightarrow$ HCb-ACEA

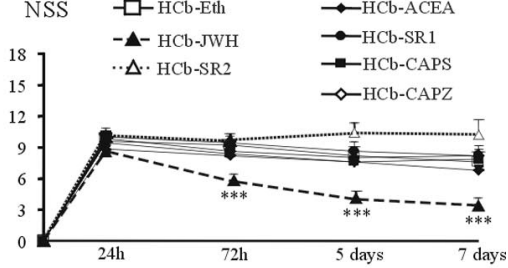

D $\triangle \mathrm{NSS}$

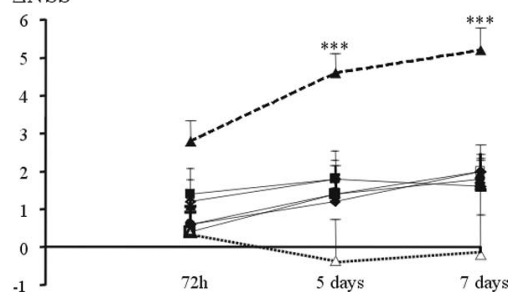

Figure 2. Effects of treatment with selective $C B 1 R, C B 2 R$, and TRPV1 agonists or antagonists on neuronal survival $(\boldsymbol{A})$ and NSS $(\boldsymbol{B}-\boldsymbol{D})$. Data are reported as means \pm SD $(n=5$ animals per group). ${ }^{* *} p<0.0001$.

treatment) that was performed on the $\Delta$ NSSs obtained from all treated HCbed groups showed a significant overall effect of treatment throughout the time course $(F=36.70$; $p<0.0001)$, but not for time $(F=4 ; p=0.07)$ nor for their interaction $(F=4.39$; $p=0.87)$. Post hoc comparisons demonstrated that the $\mathrm{HCb}-$ JWH group was significantly different at 5 and $7 \mathrm{~d}$ from $\mathrm{HCb}-\mathrm{Eth}$, HCb-SR1, HCb-ACEA, HCb-Caps, and HCb-Capz groups $(p<$ 0.01 ). The comparison between $\mathrm{HCb}-\mathrm{JWH}$ and $\mathrm{HCb}-\mathrm{SR} 2$ data also was significant $(p<0.001)$ at both time points (Fig. $2 D)$.

Cytochrome- $c($ cyt $-c)$ release from mitochondria is an early marker of apoptosis (Springer et al., 1999; Vanderluit et al., 2003). We investigated the possible effects of CB2R modulation on the apoptotic cascade by analyzing cyt- $7 \mathrm{~d}$ after $\mathrm{HCb}$. We observed self-evident differences in cyt-c immunostaining patterns between CTRL-Eth and HCb-Eth animals, as well as between $\mathrm{HCb}-\mathrm{JWH}$ and $\mathrm{HCb}$-Eth or between $\mathrm{HCb}-\mathrm{JWH}$ and $\mathrm{HCb}$ SR2 (Fig. 3A).

Quantitative analyses confirmed that JWH-015 treatment significantly reduced the percentage of neurons that released cyt- $c$ (Fig. 3B). One-way ANOVA, performed on the percentage of 
A

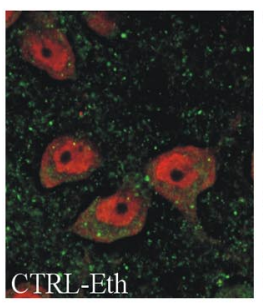

$\mathrm{C}$

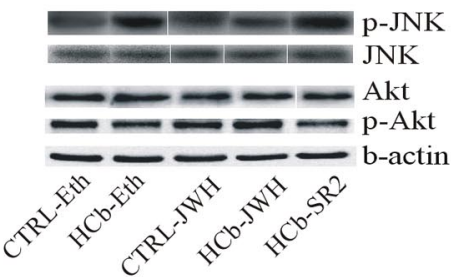

cyt-c + NeuN

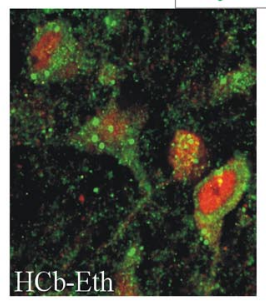

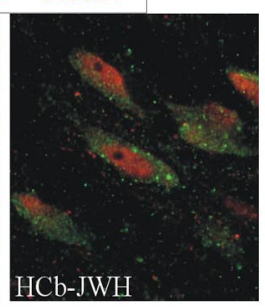

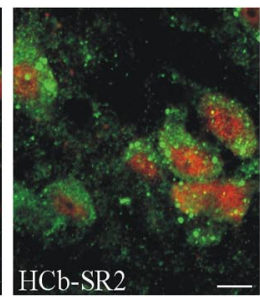

$\mathrm{B}$

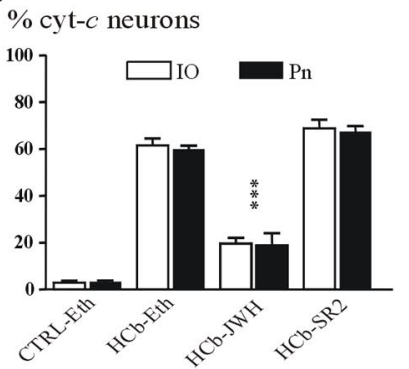

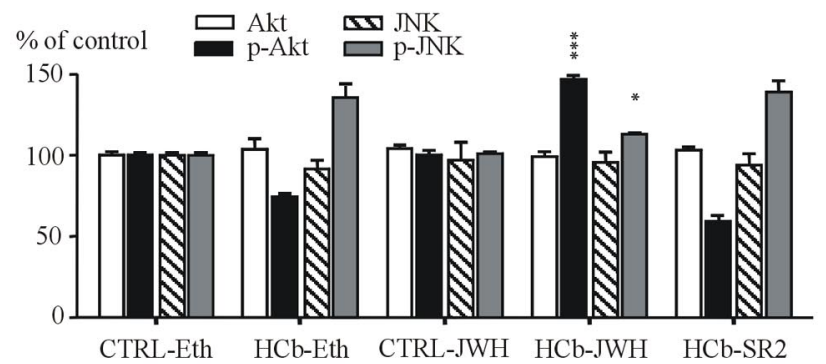

$\mathrm{D}$

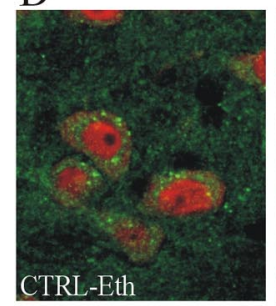

$\mathrm{F}$

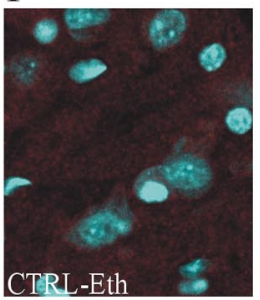

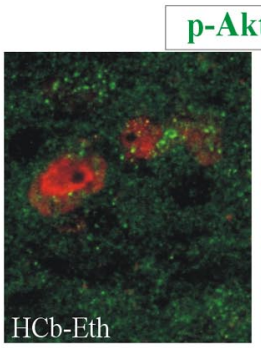

p-Akt + NeuN

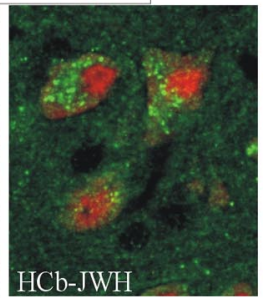

p-JNK + Dapi
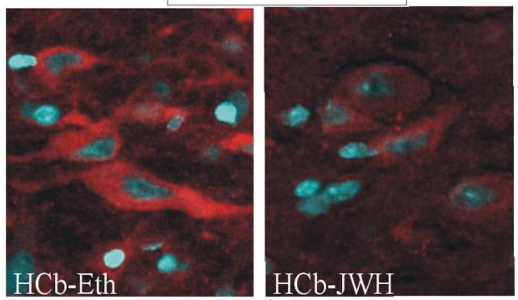
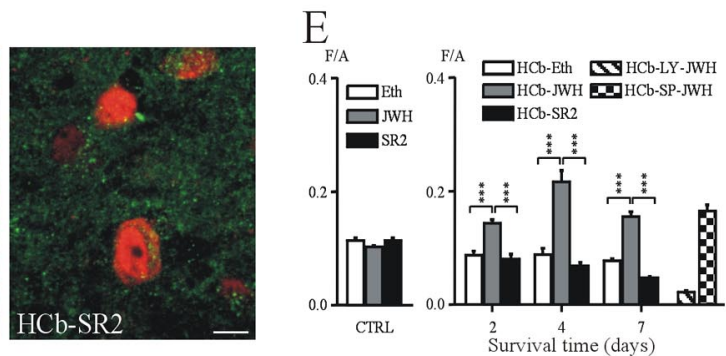

G

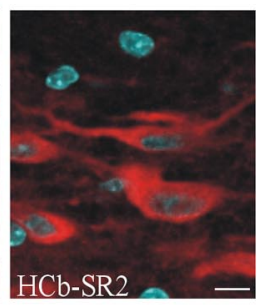

Figure 3. JWH- 015 treatment modulates cytochrome-c (cyt-c) release and p-Akt and p-JNK expression in HCb animals. $A$, cyt-c and NeuN double-labeling confocal images from Pn of CTRL-Eth, $\mathrm{HCb}$-Eth, HCb-SR2, and HCb-JWH animals. B, Percentages of NeuN-positive neurons releasing cyt-c in the 10 and Pn in CTRL-Eth, HCb-Eth, HCb-JWH, and HCb-SR2 animals. C, Western blot analysis of Akt, p-Akt, JNK, and p-JNK proteins. Band levels (left) and densitometric values (right) are shown. ${ }^{* *} \mathrm{HCb}-J W H$ versus HCb-HCb-Eth and HCb-JWH versus HCb-SR2 (p-Aht values); ${ }^{*} H C b-J W H$ versus HCb-HCb-Eth and HCb-JWH versus HCb-SR2 (p-JNK values). D, p-Akt and NeuN double-labeling confocal images from Pn of CTRL-Eth, HCb-Eth, HCb-JWH, and HCb-SR2 rats. E, Time course of densitometric values of p-Akt expressed as mean fluorescence of individual cells normalized to total cellular surface (F/A) in CTRL-Eth, CTRL-JWH, CTRL-SR2, HCb-Eth, HCb-JWH, HCb-SR2, HCb-LY-JWH, and HCb-SP-JWH rats. F, Confocal images from Pn of CTRL-Eth, HCb-Eth, HCb-JWH, and HCb-SR2 rats reacted with p-JNK antibody and counterstained with DAPI. G, Time course of densitometric values of $p$-JNK expressed as mean fluorescence of individual cells normalized to total cellular surface (F/A) in CTRL-Eth, CTRL-JWH, CTRL-SR2, HCb-Eth, HCb-JWH, HCb-SR2, HCb-LY-JWH, and HCb-SP-JWH rats. $B, C, E, G$, Data are reported as means \pm SD $\left(n=5\right.$ animals per group). ${ }^{*} p<0.05 ;{ }^{* * *} p<0.001$. Scale bars: $8 \mu \mathrm{m}$.

neurons that released cyt-c in $\mathrm{HCb}-\mathrm{JWH}, \mathrm{HCb}-\mathrm{Eth}$ and $\mathrm{HCb}-$ SR2 animals, showed a significant overall effect of the treatment (IO: $F=137.86, p<0.0001$; $\mathrm{P}: F=103.64, p<0.0001$ ). Post hoc comparisons demonstrated that our $\mathrm{HCb}-\mathrm{JWH}$ data were significantly different from those of the HCb-Eth (IO, Pn: $p<0.001$ ) and HCb-SR2 (IO, Pn: $p<0.001$ ) groups. No significant differences were observed between the $\mathrm{HCb}$-Eth and $\mathrm{HCb}-\mathrm{SR} 2$ groups (Fig. 3B).

Further, we investigated the intracellular signaling pathways that underlie the neuroprotective action of CB2R. In various cell death paradigms, CBRs can trigger phosphorylation cascades that involve mitogen-activated protein kinases (including
ERK1/2 and p38), phosphoinositide 3-kinase PI3K/Akt, and c-Jun N-terminal kinase (JNK) signaling (Derkinderen et al., 2003; Sarker et al., 2003; Molina-Holgado et al., 2005). Thus, we evaluated the phosphorylation status of ERK1/2, p38, JNK, and $\mathrm{PI} 3 \mathrm{~K} / \mathrm{Akt}$ in control and lesioned animals that were treated with ethanol or with a CB2R agonist or an antagonist.

CTRL-Eth and $\mathrm{HCb}-$ Eth animals did not show any significant difference in the total level of ERK1/2, p38, Akt, and JNK (Fig. 3C; supplemental Fig. 2A, $B$, available at www.jneurosci.org as supplemental material). The phosphorylation of ERK1/2 and p38 was not affected by the lesion (supplemental Fig. $2 A-B$, available at www.jneurosci.org as supplemental material), while both Akt 
and JNK phosphorylation were affected (Fig. $3 C, D, F)$. In lesioned animals, CB2R agonist and antagonist did not alter ERK1/2 or p38 phosphorylation (supplemental Fig. $2 A, B$, available at www.jneurosci.org as supplemental material) but they were active on Akt and JNK phosphorylation. JWH-015 increased phosphorylated Akt ( $\mathrm{p}$-Akt) and reduced phosphorylated JNK (p-JNK), while SR144528 reduced p-Akt and increased p-JNK (Fig. 3C,D,F).

Consistent with the low CB2R expression that is observed under physiological conditions, JWH-015 treatment did not modify p-Akt or p-JNK levels in control animals (Fig. 3C; compare CTRL-Eth and CTRL-JWH). One-way ANOVA of the p-Akt or of the p-JNK values showed a significant effect of the treatment ( $\mathrm{p}$-AKT: $F=244.48, p<0.0001 ;$ p-JNK: $F=7.86$, $p<0.05)$. Post hoc comparisons demonstrated that p-Akt or p-JNK levels of CTRL-Eth animals were significantly different from the HCb-Eth (p-Akt: $p<0.01$; p-JNK: $p<0.01), \mathrm{HCb}-J W H$ (p-Akt: $p<$ 0.001 ; p-JNK: $p<0.05$ ), and HCb-SR2 (pAkt: $p<0.001$; $\mathrm{p}-\mathrm{JNK}: p<0.01)$ groups. Furthermore, $\mathrm{p}$-Akt and $\mathrm{p}$-JNK values of the $\mathrm{HCb}-\mathrm{JWH}$ group were significantly different from $\mathrm{HCb}$-Eth ( $\mathrm{p}$-Akt: $p<0.001$; p-JNK: $p<0.05$ ) and HCb-SR2 (p-Akt: $p<0.001$; $\mathrm{p}$-JNK: $p<0.05)$ animals. No significant differences were observed between the $\mathrm{HCb}$-Eth and $\mathrm{HCb}-\mathrm{SR} 2$ groups in either p-Akt or p-JNK values (Fig. 3C).

To determine the role of PI3K or JNK signaling in $\mathrm{CB} 2 \mathrm{R}$ stimulation effects, we irreversibly inhibited PI3K or JNK by injecting LY294002 or SP600125, respectively, into the right lateral ventricle $90 \mathrm{~min}$ before $\mathrm{HCb}$. In addition, the animals were treated with JWH-015 [HCb-LY-JWH and HCb-SP-JWH groups (Table 1)] or ethanol [HCb-LY-Eth and HCb-SP-Eth groups (Table 1)]. Immunostaining revealed that LY294002 completely abolished JWH-015-induced p-Akt expression, while it significantly increased p-JNK expression (Fig. 3E). SP600125 treatment almost completely abolished p-JNK expression with no evident effects on JWH-015-induced p-Akt expression (Fig. 3G).

Blockade of p-Akt also inhibited JWH-015-mediated protection of neuronal survival, cyt-c release, and neurological improvement (Fig. 4A-C, see group HCb-LY-JWH). Conversely, inhibition of p-JNK did not significantly affect JWH-015mediated neuroprotection as evaluated by neuronal survival, cyt $c$ c release, and neurological improvement (Fig. 4A-C, see group $\mathrm{HCb}-\mathrm{SP}-\mathrm{JWH}$ ). One-way ANOVA demonstrated highly significant effect of treatment on neuronal survival (IO $F=59.9$, $p<0.0001$; Pn $F=31.6, p<0.0001$ ) and cyt-c release (IO $F=$ 88.5, $p<0.0001$; Pn $F=68.1, p<0.0001$ ). As for neurological improvement, two-way ANOVA, time $\times$ treatment, demonstrated a highly significant effect of time $(F=125.4, p<0.0001)$ and treatment $(F=9.2, p<0.0001)$. Interaction was not significant. Post hoc comparisons are detailed in supplemental Table 1 (available at www.jneurosci.org as supplemental material).

Taking into account the interrelations between CB2R signal- ing and p-Akt or p-JNK and the known importance of JNK in signal transduction after axotomy (Herdegen and Waetzig, 2001), we analyzed by quantitative densitometry the time course of p-Akt and p-JNK levels (day 0 to day 7 after $\mathrm{HCb}$ ) in Pn and their modulation by CB2R signaling (Fig. $3 E, G$ ). HCb-Eth animals presented a small progressive reduction of $\mathrm{p}$-Akt with a clear increment, especially after day 4 , of p-JNK. CB2R stimulation by JWH-015 reversed the effect of the lesion on p-Akt, starting from day 2 with the highest peak at day 4 . As for p-JNK, CB2R stimulation significantly reduced the lesion-induced increase throughout the observed time window. The CB2R antagonist SR144528 slightly enhanced the lesion-induced reduction in p-Akt while it did not influence the p-JNK time course. Two-way ANOVA (time $\times$ treatment) that was performed on the $\mathrm{p}$-Akt and $\mathrm{p}$-JNK F/A index of $\mathrm{HCb}-\mathrm{Eth}, \mathrm{HCb}-\mathrm{JWH}$, and $\mathrm{HCb}-\mathrm{SR} 2$ groups at the time points at 2, 4, and $7 \mathrm{~d}$ after lesion showed a significant overall effect of treatment (p-Akt: $F=196.8$; $p<0.0001$; p-JNK: $F=$ 38.97; $p<0.0001$ ) and of time (p-Akt: $F=5.43$; $p<0.01$; $\mathrm{p}-\mathrm{JNK}$ : $F=329.8 ; p<0.0001)$. Also, interaction was significant (p-Akt: $F=7.68 ; p<0.0001$; p-JNK: $F=51.9 ; p<0.0001)$. Post hoc comparisons are reported in Figure 3, $E$ and $G$.

\section{Discussion}

This study demonstrates the induction of $\mathrm{CB} 2 \mathrm{R}$ in axotomized central neurons and the protective effect of the CB2R selective 
agonist JWH-015 on neuronal survival and functional recovery. Furthermore, our study of CB2R signaling indicates that CB2Rrelated neuroprotection involves the PI3K/Akt pathway.

The presence of CB2R in the brain is controversial, particularly with regard to its expression in central neurons (Van Sickle et al., 2005; Chin et al., 2008). Our immunohistochemistry and qPCR data confirmed the absence or low expression of CB2R in the brain under normal conditions, at least for the two structures that we considered-the IO and Pn. Remarkably, axonal lesions effected an increase in CB2R expression in approximately half of all lesioned neurons. This observation was corroborated by our immunohistochemistry (Fig. 1 $A, B$ ) and qPCR (Fig. 1D) data.

The functional significance of this postlesional change in CBR expression pattern in axotomized neurons was analyzed by evaluating the effects of selective agonists and antagonists of CBRs on cell survival and functional recovery. Treatment with capsaicin or capsazepine, a TRPV1-selective agonist and antagonist, respectively (Starowicz et al., 2007), or with a CB1R selective agonist, ACEA, and antagonist, SR141716A (Mackie, 2006), did not affect lesion-induced neuronal loss or postlesional neurological status. In this context, it should be recalled that TRPV1 has been shown to mediate endocannabinoid-induced apoptosis in different in vitro models (Maccarrone, 2006). On the other hand, the selective CB2R agonist JWH-015 improved both cell survival and functional recovery (Fig. 2A-D).

Interestingly, JWH-015 and the $\mathrm{CB} 2 \mathrm{R}$ selective antagonist SR144528 had opposite effects. As shown in Figure 2A, the number of neurons in the IO or Pn in unlesioned animals was comparable across all groups, independent of the treatment with various CBR-related compounds, thus confirming the efficacy of the quantitative methods that were used. With the exception of those that were treated with a $\mathrm{CB} 2 \mathrm{R}$ agonist or antagonist, all groups of HCbed rats experienced the same degree of neuronal loss as the vehicle-treated group. Only CB2R modulation was effective in improving cell survival. Indeed, the CB2R agonist enhanced the number of surviving neurons, while the $\mathrm{CB} 2 \mathrm{R}$ antagonist tended to reduce it.

The specificity of CB2R modulation was confirmed by our NSS data (Fig. 2B-D). Also, in these analyses, the only compounds that affected neurological recovery were those that activated $\mathrm{CB} 2 \mathrm{R}$. In unlesioned animals, none of the compounds influenced the NSS. All groups generated the same NSS after the first $24 \mathrm{~h}$ after $\mathrm{HCb}$. At subsequent points, all groups had steady profiles, with the exception of animals that were treated with JWH-015 or SR144528. The most profound changes were observed in the CB2R agonist-treated group, which was the only one that showed progressive improvement in neurological performance. Rats that were treated with the CB2R antagonist showed a decline in NSS that, nevertheless, did not differ significantly from that of vehicle-treated rats. The efficacies and the opposing patterns of the CB2R agonist and antagonist were further corroborated by the $\Delta$ NSS values (Fig. $2 D$ ).

Cell death of precerebellar neurons after $\mathrm{HCb}$ is associated with necrosis and apoptosis (Viscomi et al., 2004). Immunohistochemical detection of cyt-c release is considered to be an early indication of apoptosis (Vanderluit et al., 2003), as well as endocannabinoid-induced apoptosis (Bari et al., 2005). Here, we showed that $\mathrm{HCb}$ induced cyt-c release in $\sim 60 \%$ of surviving precerebellar neurons, while JWH-015 reduced this percentage to $20 \%$. Conversely, treatment with SR144528 increased the number of neurons that released cyt-c (Fig. $3 A, B$ ).

We propose that the expression of $\mathrm{CB} 2 \mathrm{R}$ in axotomized central neurons is a neuroprotective mechanism since its stimulation reduces cell death by blocking cyt-c-associated apoptosis. Establishing the link between sparing neuronal death and improving functional recovery is challenging; nevertheless, it requires further investigation because CB2R activity may affect NSS by acting on different neural centers from those that were considered herein. At any rate, the present data demonstrate that CB2R activation protects cells against apoptotic death, extending and applying previous data from different in vitro paradigms to an in vivo system (Maccarrone, 2006).

Recent studies have implicated CB2R in the neuroprotective activity of cannabinoids, which act primarily through a series of glia-dependent anti-inflammatory processes (Fernández-Ruiz et al., 2007). In various inflammatory paradigms, CB2R is upregulated in reactive microglial cells and astrocytes in response to inflammatory stimuli. In the present study, CB2R was not expressed on glial cells (supplemental Fig. 1, available at www. jneurosci.org as supplemental material), arguing against the hypothesis that modulation of $\mathrm{CB} 2 \mathrm{R}$ expression occurs predominantly in glial cells during remote cell death.

The principal signal transduction pathways that are associated with postlesional stimulation of CB2R were analyzed by modulating PI3K, ERK1/2, p38, and JNK. HCb or JWH-015 treatment did not alter activity of the ERK1/2 or 38 pathways (supplemental Fig. 2, available at www.jneurosci.org as supplemental material). Conversely, $\mathrm{HCb}$ induced a reduction in p-Akt and an increase in p-JNK. CB2R modulation selectively changed postlesional PI3K/Akt and JNK signaling (Fig. 3C-G). Namely, CB2R agonist treatment counteracted the lesion-induced effects by enhancing p-Akt and decreasing p-JNK, while CB2R antagonist treatment tended to worsen the lesion effects further reducing p-Akt values (Fig. 3C-G). The other two downstream pathways of CB2R (ERK1/2 or p38) (supplemental Fig. 2, available at www.jneurosci.org as supplemental material) were not affected by CB2R stimulation.

The importance of PI3K/Akt signaling in JWH-015-mediated neuroprotection was further highlighted by treatment with a combination of both PI3K inhibitor (LY294002) or JNK inhibitor (SP600125) and JWH-015. Pretreatment with LY294002 abolished all JWH-015-induced indications of neuroprotection-namely, p-Akt expression, cell survival, reduction of cyt- $c$ release, and NSS improvement. Conversely, JNK blockade did not reduce the JWH-015 neuroprotective effects (Fig. 4A-D).

In conclusion, our data favor the model (depicted in supplemental Fig. 3, available at www.jneurosci.org as supplemental material) wherein CB2R activation counteracts apoptotic cell death that is induced by remote axonal damage by acting through PI3K/Akt signaling. This is the first report that describes gliaindependent protection by $\mathrm{CB} 2 \mathrm{R}$ within the brain in vivo. This unprecedented evidence also extends the concept that cannabinoid receptor activation has anti-apoptotic effects to an in vivo system, which, until now, had been documented only in in vitro models.

\section{References}

Bari M, Battista N, Fezza F, Finazzi-Agrò A, Maccarrone M (2005) Lipid rafts control signaling of type- 1 cannabinoid receptors in neuronal cells. Implications for anandamide-induced apoptosis. J Biol Chem 280:12212-12220.

Binkofski F, Seitz RJ, Arnold S, Classen J, Benecke R, Freund HJ (1996) Thalamic metbolism and corticospinal tract integrity determine motor recovery in stroke. Ann Neurol 39:460-470.

Block F, Dihné M, Loos M (2005) Inflammation in areas of remote changes following focal brain lesion. Prog Neurobiol 75:342-365.

Centonze D, Finazzi-Agrò A, Bernardi G, Maccarrone M (2007) The endo- 
cannabinoid system in targeting inflammatory neurodegenerative diseases. Trends Pharmacol Sci 28:180-187.

Chin CL, Tovcimak AE, Hradil VP, Seifert TR, Hollingsworth PR, Chandran P, Zhu CZ, Gauvin D, Pai M, Wetter J, Hsieh GC, Honore P, Frost JM, Dart MJ, Meyer MD, Yao BB, Cox BF, Fox GB (2008) Differential effects of cannabinoid receptor agonists on regional brain activity using pharmacological MRI. Br J Pharmacol 153:367-379.

Derkinderen P, Valjent E, Toutant M, Corvol JC, Enslen H, Ledent C, Trzaskos J, Caboche J, Girault JA (2003) Regulation of extracellular signalregulated kinase by cannabinoids in hippocampus. J Neurosci 23:2371-2382.

Di Marzo V, Bisogno T, De Petrocellis L (2007) Endocannabinoids and related compounds: walking back and forth between plant natural products and animal physiology. Chem Biol 14:741-756.

Fernández-Ruiz J, Romero J, Velasco G, Tolón RM, Ramos JA, Guzmán M (2007) Cannabinoid CB2 receptor: a new target for controlling neural cell survival? Trends Pharmacol Sci 28:39-45.

Herdegen T, Waetzig V (2001) The JNK and p38 signal transduction following axotomy. Restor Neurol Neurosci 19:29-39.

Klein TW, Newton C, Larsen K, Lu L, Perkins I, Nong L, Friedman H (2003) The cannabinoid system and immune modulation. J Leukoc Biol 74:486-496.

Maccarrone M (2006) Involvement of the endocannabinoid system in cancer. In: Endocannabinoids: the brain and the body's marijuana and beyond (Onaivi ES, Sugiura T, Di Marzo V, eds), pp 451-466. Boca Raton, FL: CRC/Taylor and Francis.

Maccarrone M, Finazzi-Agró A (2003) The endocannabinoid system, anandamide and the regulation of mammalian cell apoptosis. Cell Death Differ 10:946-955.

Mackie K (2006) Cannabinoid receptors as therapeutic targets. Annu Rev Pharmacol Toxicol 46:101-122.

Molina-Holgado F, Pinteaux E, Heenan L, Moore JD, Rothwell NJ, Gibson RM (2005) Neuroprotective effects of the synthetic cannabinoid HU210 in primary cortical neurons are mediated by phosphatidylinositol 3-kinase/AKT signaling. Mol Cell Neurosci 28:189-194.
Onaivi ES, Ishiguro H, Gong JP, Patel S, Perchuk A, Meozzi PA, Myers L, Mora Z, Tagliaferro P, Gardner E, Brusco A, Akinshola BE, Liu QR, Hope B, Iwasaki S, Arinami T, Teasenfitz L, Uhl GR (2006) Discovery of the presence and functional expression of cannabinoid CB2 receptors in brain. Ann N Y Acad Sci 1074:514-536.

Sarker KP, Biswas KK, Yamakuchi M, Lee KY, Hahiguchi T, Kracht M, Kitajima I, Maruyama I (2003) ASK1-p38 MAPK/JNK signaling cascade mediates anandamide-induced PC12 cell death. J Neurochem 85:50-61.

Springer JE, Azbill RD, Knapp PE (1999) Activation of the caspase-3 apoptotic cascade in traumatic spinal cord injury. Nat Med 5:943-946.

Starowicz K, Nigam S, Di Marzo V (2007) Biochemistry and pharmacology of endovanilloids. Pharmacol Ther 114:13-33.

Stella N (2004) Cannabinoid signaling in glial cells. Glia 48:267-277.

Vanderluit JL, McPhail LT, Fernandes KJ, Kobayashi NR, Tetzlaff W (2003) In vivo application of mitochondrial pore inhibitors blocks the induction of apoptosis in axotomized neonatal facial motoneurons. Cell Death Differ 10:969-976.

Van Sickle MD, Duncan M, Kingsley PJ, Mouihate A, Urbani P, Mackie K, Stella N, Makriyannis A, Piomelli D, Davison JS, Marnett LJ, Di Marzo V, Pittman QJ, Patel KD, Sharkey KA (2005) Identification and functional characterization of brainstem cannabinoid CB2 receptors. Science 310:329-332.

Viscomi MT, Florenzano F, Conversi D, Bernardi G, Molinari M (2004) Axotomy dependent purinergic and nitrergic co-expression. Neuroscience 123:393-404.

Viscomi MT, Florenzano F, Latini L, Amantea D, Bernardi G, Molinari M (2008) Methylprednisolone treatment delays remote cell death after focal brain lesion. Neuroscience 154:1267-1282.

Watanabe T, Okuda Y, Nonoguchi N, Zhao MZ, Kajimoto Y, Furutama D, Yuhawa H, Shibata MA, Otsuki Y, Kuroiwa T, Miyatake S (2004) Postischemic intraventricular administration of FGF-2 expressing adenoviral vectors improves neurologic outcome and reduces infarct volume after transient focal cerebral ischemia in rats. J Cereb Blood Flow Metab 24: $1205-1213$. 